\title{
Spintronic terahertz-frequency nonlinear emitter based on the canted antiferromagnet-platinum bilayers
}

Cite as: J. Appl. Phys. 125, 223903 (2019); doi: 10.1063/1.5090455

Submitted: 28 January 2019 . Accepted: 27 May 2019.

Published Online: 12 June 2019

P. Stremoukhov, ${ }^{1,2, a)}$ (D) A. Safin, ${ }^{3,4}$ (D) M. Logunov, ${ }^{3}$ S. Nikitov, ${ }^{2,3}$ and A. Kirilyuk $^{7}$ (D)

\author{
AFFILIATIONS \\ ${ }^{7}$ FELIX Laboratory, Radboud University, Toernooiveld 7c, 6525 ED Nijmegen, The Netherlands \\ ${ }^{2}$ Moscow Institute of Physics and Technology, Dolgoprudny, Moscow Region 141700, Russia \\ ${ }^{3}$ Kotel'nikov Institute of Radioengineering and Electronics, Russian Academy of Sciences, 125009 Moscow, Russia \\ ${ }^{4}$ National Research University "Moscow Power Engineering Institute," 111250 Moscow, Russia
}

Note: This paper is part of the Special Topic section "Advances in Terahertz Solid-State Physics and Devices" published in J. Appl. Phys. 125(15) (2019).

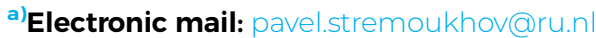

\begin{abstract}
In this paper, we propose an approximate nonlinear theory of a spintronic terahertz-frequency emitter based on canted antiferromagnetplatinum bilayers. We present a model accounting for the excitation of nonlinear oscillations of the Néel vector in an antiferromagnet using terahertz pulses of an electromagnetic field. We determine that, with increasing amplitude of the pumping pulse, the spin system's response increases nonlinearly in the fundamental quasiantiferromagnetic mode. We demonstrate control of the Néel vector trajectory by changing the terahertz pulse peak amplitude and frequency and determine the bands of nonlinear excitation using Fourier spectra. Finally, we develop an averaging method which gives the envelope function of an oscillating output electromagnetic field. The nonlinear dynamics of the antiferromagnet-based emitters discussed here is of importance in terahertz-frequency spintronic technologies.
\end{abstract}

Published under license by AIP Publishing. https://doi.org/10.1063/1.5090455

\section{INTRODUCTION}

Antiferromagnetic spintronics is an emerging field of magnetism where several fundamental discoveries have been made in the past few years, ${ }^{1-3}$ resulting in novel technological ideas. ${ }^{4,5}$ The key goal of antiferromagnetic spintronics is to demonstrate devices that enable information processing and storage on the terahertzfrequency scale. The latest advances have thus made it possible to observe and control low-energy excitations on picosecond and femtosecond timescales. ${ }^{1,6,7}$

Until recently, antiferromagnets were considered as theoretically interesting but still without any practical applications. Nevertheless, the dynamics of spin order in antiferromagnets were shown to be intrinsically ultrafast ${ }^{8-10}$ (see the overview in Ref. 11 for more details). These results unlock a multitude of known and newly identified unique features of antiferromagnets relevant for applications in spintronics. ${ }^{11}$ Experimentally, terahertz excitation of antiferromagnetic resonance was shown for $\mathrm{NiO} .^{2,12}$ Moreover, it has recently been proposed and demonstrated that when driving a macroscopic electrical current through certain antiferromagnetic crystals (e.g., CuMnAs, $\mathrm{Mn}_{2} \mathrm{Au}$ ), a fieldlike Néel spin-orbit torque emerges that allows for a reversible switching of antiferromagnetic moments. ${ }^{10,13}$ Metallic CuMnAs and insulating $\mathrm{NiO}$ mentioned above are examples of the simplest two-spin-sublattice collinear antiferromagnets.

Recently, it was reported that terahertz-frequency emission can be realized in nanosized structures composed of heavy metal (HM) and ferromagnetic (FM) thin films upon excitation of the latter by short laser pulses. ${ }^{14-16}$ The transient currents are generated via the inverse spin Hall effect (ISHE) on the spin current injected into the HM film from the demagnetized FM film. Because the AFM and HM layers have different transport properties, a net current along the $z$-axis is launched. In addition, the product of the density, band-velocity, and lifetime of spin-up (majority) electrons, which 
is significantly higher than that of the spin-down (minority) electrons, is strongly spin-polarized. ${ }^{17}$ On entering the HM layer, spin-orbit coupling deflects the spin-up and spin-down electrons in opposite directions. ${ }^{18}$ This ISHE converts the longitudinal spin current density into a transverse charge current density. The detection of the net spin current flowing into the HM can be achieved electrically via the ISHE, as was demonstrated before. ${ }^{18}$ Such generation of a transverse electric current by a spin current injected into a paramagnetic metal can also be employed to detect the effect of spin pumping, ${ }^{19}$ resulting from the excitation of ferromagnetic or antiferromagnetic resonances. ${ }^{20}$

The FM material in such experiments is usually magnetized by an applied magnetic field, which also sets the frequency of the excitation (ferromagnetic resonance). One of the possible ways to develop magnetic-based terahertz emitters without applied magnetic fields is to use AFM materials with strong internal magnetic fields (originating from, e.g., exchange magnetic field between sublattices). A detailed theoretical study of the electric field arising due to the ISHE in a nonmagnetic metal resulting from the spin current from an antiferromagnet is presented in Refs. 21 and 22. We use the results obtained in these papers to calculate the output electric field generated by the AFM emitter. While the terahertz-driven nonlinear spin response of a thin AFM film was discovered in Ref. 3, the understanding of the nonlinear ultrafast processes in antiferromagnets is still in its infancy.

In this work, we propose and theoretically analyze a terahertzfrequency nonlinear emitter based on the canted AFM (taking hematite as an example) and HM (such as platinum). The article is organized as follows. In Sec. II, we consider a physical structure of the AFM-based terahertz-frequency nonlinear emitter. Then, we present (Sec. III) a model of excitation of nonlinear spin oscillations of the Néel vector in an antiferromagnet under the action of terahertz pulses delivered by an electromagnetic field. We demonstrate the control of the Néel vector trajectory by changing the terahertz pulse peak amplitude and frequency. In Sec. IV, we develop the averaging method, which gives the envelope function of an oscillating output electromagnetic field. Finally, Sec. $V$ is devoted to numerical simulations used to determine the bands of nonlinear excitations using Fourier spectra. Because of the ISHE, we find that increasing the pumping pulse amplitude causes the spin system's response amplitude to increase nonlinearly in the fundamental quasiantiferromagnetic mode.

\section{PHYSICAL STRUCTURE}

Figure 1 schematically shows a bilayered AFM-based terahertz emitter consisting of a $\mathrm{Pt} / \alpha-\mathrm{Fe}_{2} \mathrm{O}_{3}$ under photoexcitation. A terahertz laser pulse pumps the AFM resonance mode of $\alpha-\mathrm{Fe}_{2} \mathrm{O}_{3}$ and, via spin pumping, drives a spin current into the HM layer. The ISHE transforms the spin current into a picosecond pulse of transverse charge current. As a result, an electromagnetic wave with near-terahertz frequency (depending specifically on the pulse's temporal profile) is produced by the transient charge current. This terahertz pulse is radiated out of the film plane, with its polarization being set by the direction of the electric current. Here, we do not take into account any externally applied constant magnetic field, which could reorient the magnetic sublattices in the AFM. Since an

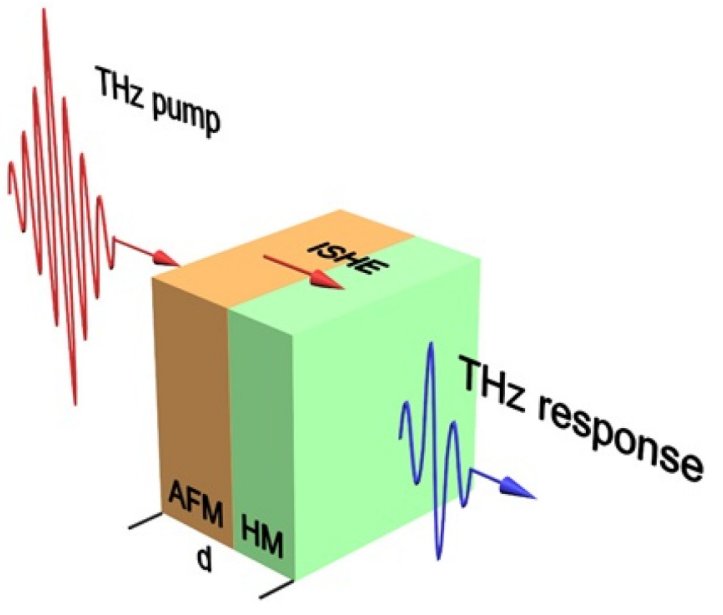

FIG. 1. Schematic view of the terahertz-frequency AFM-based emitter. A terahertz pulse pumps an AFM/HM heterostructure of thickness $d$ and generates nonequilibrium pure spin current injected into the HM layer. The spin current is converted into a transient charge current due to the inverse spin Hall effect (ISHE) in the HM layer. This charge current generates the outgoing terahertz pulse.

external field is not applied to the sample, the two-magnon modes in the AFM are degenerate. ${ }^{23}$

We consider hematite $\alpha$ - $\mathrm{Fe}_{2} \mathrm{O}_{3}$ as a prototypical example of an AFM. The bulk Dzyaloshinskii-Moriya interaction (DMI) ${ }^{24,25}$ inside the AFM layer leads to the canting of the magnetization $\mathbf{M}_{1}$ and $\mathbf{M}_{2}$ within the AFM sublattices, thus creating a small net magnetization. Once excited by terahertz laser pulse, the magnetization of each sublattice $\mathbf{M}_{\mathbf{1}}$ and $\mathbf{M}_{\mathbf{2}}$ exhibits periodical precessionlike dynamics. The directions of the magnetization vectors and the anisotropy axes, with respect to the sample geometry, are shown in Fig. 2.

Figure 3(a) shows the typical pulse with pumping frequency $\Omega / 2 \pi=0.265 \mathrm{THz}$ and pulse amplitude $\mu_{0} \cdot H_{\max }=0.1 \mathrm{~T}$. We set

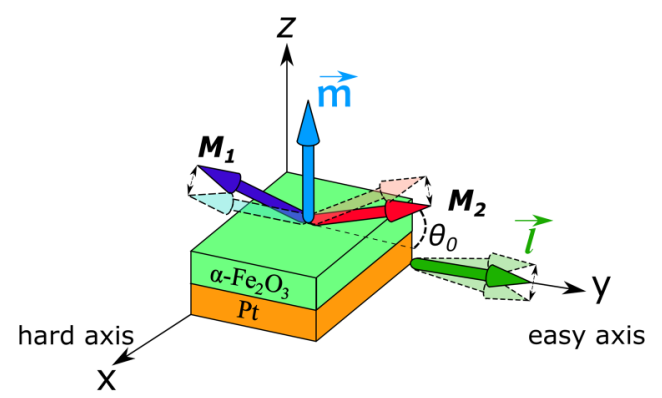

FIG. 2. Schematic representation of the rotating sublattice-specific magnetization $\mathbf{M}_{1}$ and $\mathbf{M}_{2}$ in an AFM. The presence of the easy plane magnetic anisotropy in the AFM layer leads to a variable in time rotation speed of the AFM sublattice magnetizations with oscillation frequency. The magnetization of the AFM sublattices are canted by a small angle $\theta_{0}$ due to the DMI. 

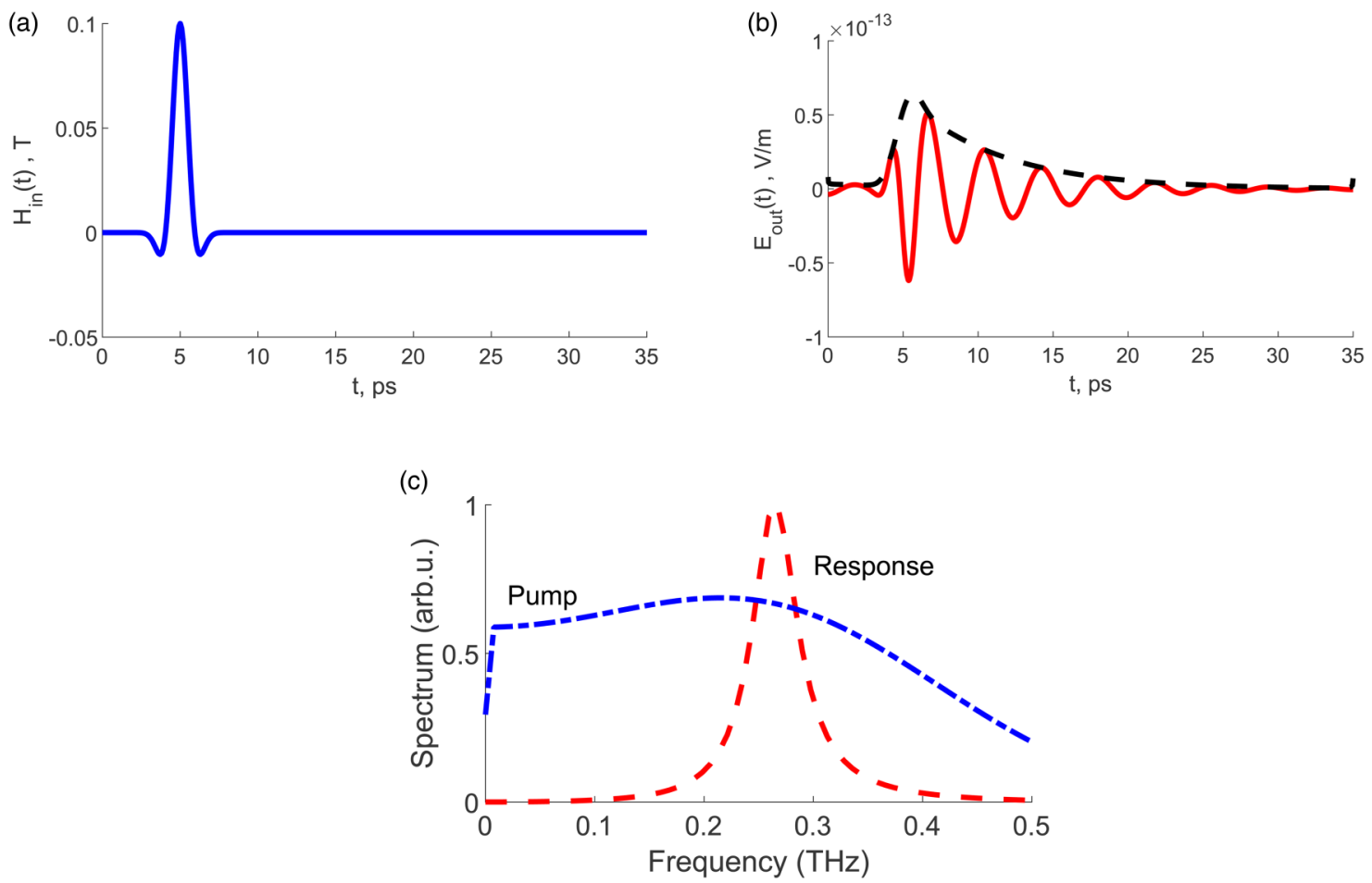

FIG. 3. (a) The profile of the pump pulse with central frequency $\Omega / 2 \pi=0.265 \mathrm{THz}$, time offset $t_{0}=5 \mathrm{ps}$, and pulse width $\tau=1$ ps. (b) Numerical solution of Eq. (2), describing the response, which is normalized to the maximum value (dashed line corresponds to the envelope function). (c) Spectra of the pump terahertz signal and output response.

the shape of the magnetic field pulse $\mathbf{H}_{i n}(t)$, which acts as a component of the effective magnetic field, using a Gaussian function with the terahertz filling and linear polarization along the $\mathbf{e}_{\mathbf{x}}$-axis,

$$
\mathbf{H}_{i n}(t)=H_{\max } \cdot \exp \left[-\left(\frac{t-t_{0}}{\tau}\right)^{2}\right] \cdot \cos \left(\Omega\left(t-t_{0}\right)\right) \mathbf{e}_{\mathbf{x}},
$$

where $t_{0}$ is the pulse envelope delay time and $\tau$ is the width of the pulse. Here, the terahertz pump pulse has a wide spectrum in the frequency domain, with a tuneable central frequency. The terahertz emitter is the oscillation system with an oscillation frequency, which is defined by the easy plane anisotropy field and exchange field.

\section{MATHEMATICAL MODEL}

We consider the spin dynamics in an AFM excited by terahertz pulses using the sigma-model widely used in the theory of antiferromagnetism. ${ }^{26,27}$ For this purpose, using the LandauLifshitz-Gilbert (LLG) equations of motion to describe the magnetization of sublattices $\mathbf{M}_{\mathbf{1}}$ and $\mathbf{M}_{2}$, we resort to the dynamic variable $\mathbf{l}=\left(\mathbf{M}_{\mathbf{1}}-\mathbf{M}_{2}\right) / 2 M_{s}$ (Néel vector), where $M_{s}$ is the saturation magnetization. We take in to account the fact that the total magnetization $\mathbf{m}=\left(\mathbf{M}_{\mathbf{1}}+\mathbf{M}_{\mathbf{2}}\right) / 2 M_{s}$ is a small value and $\mathbf{m} \ll \mathbf{l}$ (see Fig. 2). We express the dynamics of the vector $\mathbf{m}$ through the vector $\mathbf{l}$ and its time derivative in the following form:

$$
H_{e x} \mathbf{m}=\mathbf{H}_{e f f}-\mathbf{l}\left(\mathbf{H}_{e f f} \cdot \mathbf{l}\right)+\frac{1}{\gamma}\left(\frac{\partial \mathbf{l}}{\partial t} \times \mathbf{l}\right)
$$

where $\gamma$ is the gyromagnetic ratio, $H_{e x}$ is the exchange field between the sublattices, and $\mathbf{H}_{\text {eff }}$ is the effective magnetic field, which takes into account the anisotropy field $\mathbf{H}_{a n}$, DMI field $\mathbf{H}_{D M I}$, and the magnetic field of the terahertz pulse $\mathbf{H}_{i n}(t)$. Here, we do not take into account any constant applied magnetic field. The last term in (1) describes the dynamic part of the total magnetization $\mathbf{m}{ }^{28}$ We parameterize the vector $\mathbf{1}(t)$ in terms of polar $\theta(t)$ and azimuthal $\varphi(t)$ angles in the spherical coordinate system,

$$
l_{z}=\cos (\theta), \quad l_{x}=\sin (\theta) \cos (\varphi), \quad \text { and } \quad l_{y}=\sin (\theta) \sin (\varphi) .
$$

From the experimental data (see, e.g., Refs. 26 and 29), it is known that vector $\mathbf{l}$ is oriented almost at a constant angle $\theta=\theta_{0}$ with respect to the $\mathbf{e}_{\mathbf{z}}$-axis, and so the dynamics can be described using just the azimuthal angle $\varphi(t)$. By varying the Lagrangian

$$
L[\mathbf{l}]=\frac{\hbar}{2 \gamma H_{e x}}\left(\frac{\partial \mathbf{l}}{\partial t}\right)^{2}-W_{a}(\mathbf{l})-\frac{\hbar}{H_{e x}}\left(\mathbf{H}_{e f f} \cdot\left[\mathbf{l} \times \frac{\partial \mathbf{l}}{\partial t}\right]\right)
$$


over the angle $\varphi(t)$ with $\theta=$ const, we obtain the second-order equation for $\varphi(t),{ }^{26}$

$$
\begin{aligned}
& \frac{d^{2} \varphi}{d t^{2}}+\alpha \omega_{e x} \frac{d \varphi}{d t}+\frac{\omega_{0}^{2}}{2} \sin (2 \varphi)+\omega_{D M I} \cdot \gamma \cdot H_{\text {in }}(t) \cos (\varphi) \\
& =\gamma \frac{d H_{\text {in }}(t)}{d t} .
\end{aligned}
$$

Here $\alpha \approx 10^{-4}$ is the Gilbert damping constant, $\hbar$ is the reduced Planck constant, $\omega_{e x}=\gamma H_{e x}$ (where the exchange field is $H_{e x}=9 \quad \mathrm{MOe}$ ), $\omega_{D M I}=\gamma H_{D M I} \quad$ (where the DMI field is $\left.H_{D M I}=22 \mathrm{kOe}\right)$, and $\omega_{0}=\sqrt{\omega_{a} \omega_{e x}}$ is the quasiantiferromagnetic resonance frequency (where $\omega_{a}=\gamma H_{a}$ is the frequency related with the anisotropy field $H_{a}=200 \mathrm{Oe}$ ). The assumed parameter values correspond to those of hematite. ${ }^{5,30}$ The action of the optical terahertz pump pulse in our model is considered in Eq. (2) as a timevarying magnetic field, which drives the induced inertial dynamics.

The ISHE electric field in the HM is calculated using the analytical expression, ${ }^{31}$

$$
E_{\text {out }}=\theta_{S H} \frac{g_{\uparrow \downarrow} e \lambda \rho}{2 \pi d_{P t}} \tanh \left(\frac{d_{P t}}{2 \lambda}\right) \frac{d \varphi}{d t}=\kappa \cdot \frac{d \varphi}{d t},
$$

where $g_{\uparrow \downarrow}=6.9 \times 10^{14} \mathrm{~cm}^{-2}$ is the spin-mixing conductance at the $\mathrm{Pt}$-AFM interface, $\theta_{S H} \approx 0.1 \mathrm{rad}$ is the spin Hall angle in Pt, $e$ is the modulus of the electron charge, $\lambda=7.3 \mathrm{~nm}$ is the spindiffusion length in the Pt layer, $\rho=4.8 \times 10^{-7} \Omega \mathrm{m}$ is the electrical resistivity of $\mathrm{Pt}$, and $d_{P t}=20 \mathrm{~nm}$ is the thickness of the Pt layer., For the chosen parameters, $\kappa \approx 1.35 \times 10^{-9} \mathrm{~V} / \mathrm{m}(\mathrm{rad} / \mathrm{s})^{-1}$.

The presence of the DMI, which is represented by the 4 th term in Eq. (2), leads to inertial dynamics of the Néel vector in the AFM. In the inertial mechanism, during the action of the driving force, the orientation of the Néel vector is hardly changed, but it is enough to overcome the potential barrier afterward. ${ }^{9}$ On the one hand, the presence of DMI is essential for the nonlinear dependence of the oscillations on the excitation frequency. On the other hand, in its absence, the forced dynamics of the AFM is determined only by the gyroscopic mechanism $\gamma \frac{d H_{i n}(t)}{d t}$, which is too weak to give rise to oscillations on its own.

\section{AVERAGED EQUATIONS}

Figure 3(b) shows the result of solving Eqs. (2) and (3) in terms of the outgoing electric field $E_{\text {out }}$ normalized to the maximum value $E_{\text {out }}$ obtained by the numerical integration. Since the shape of the envelope function of the output electric field could be recorded using pump-and-probe experiments, we present here the method of its theoretical determination. Equation (2) corresponds to the equation of motion of a driven pendulum and can be examined using standard methods of the theory of nonlinear oscillations. We find the solution $\varphi(t)$ in the form of a quasiharmonic response at a frequency of a forced oscillation,

$$
\varphi(t)=\beta_{0}+\Omega \cdot t+\beta_{1}(t) \sin (\Omega t),
$$

where the amplitude $\beta_{1}(t)$ is slowly varying with time (accounted for by the envelope function) and $\beta_{0}$ is a certain constant phase. The solution (4) represents oscillations of the $l_{y}$ component the Néel vector.

Upon substituting Eq. (4) into Eq. (2) and decomposing the nonlinear term in Eq. (2) using a Fourier series, we obtain the following nonlinear equations characterizing $\beta_{0}$ and $\beta_{1}$ :

$$
\begin{aligned}
2 \Omega \frac{d \beta_{1}}{d t} & +\alpha \omega_{e x} \Omega \beta_{1}-\omega_{0}^{2} J_{1}\left(\beta_{1}\right)\left(J_{0}\left(\beta_{1}\right)+J_{2}\left(\beta_{1}\right)\right) \sin \left(2 \beta_{0}\right) \\
& -\omega_{D M I} \omega_{\max } J_{1}\left(\beta_{1}\right) f(t) \cos \left(\Omega t_{0}\right) \\
=\omega_{\max } & {\left[\Omega f(t) \sin \left(\Omega t_{0}\right)+f^{\prime}(t) \cos \left(\Omega t_{0}\right)\right], }
\end{aligned}
$$
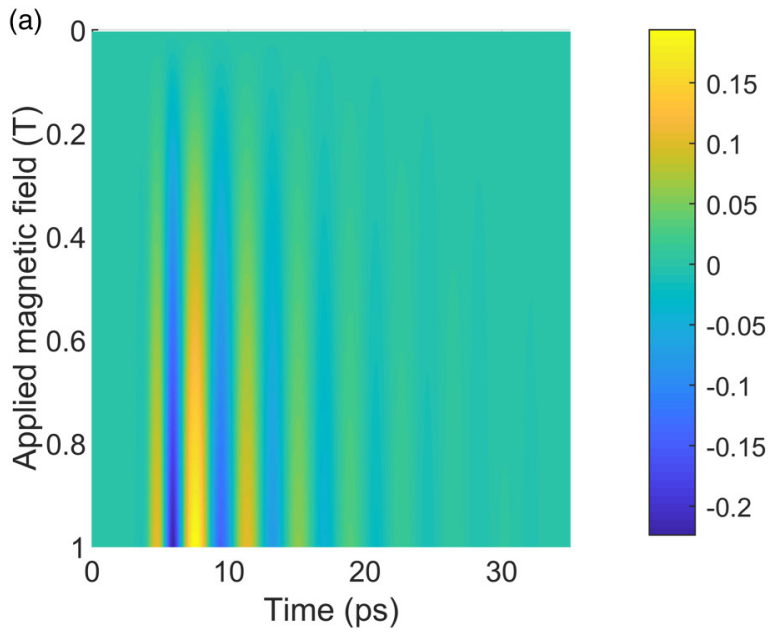

(b)

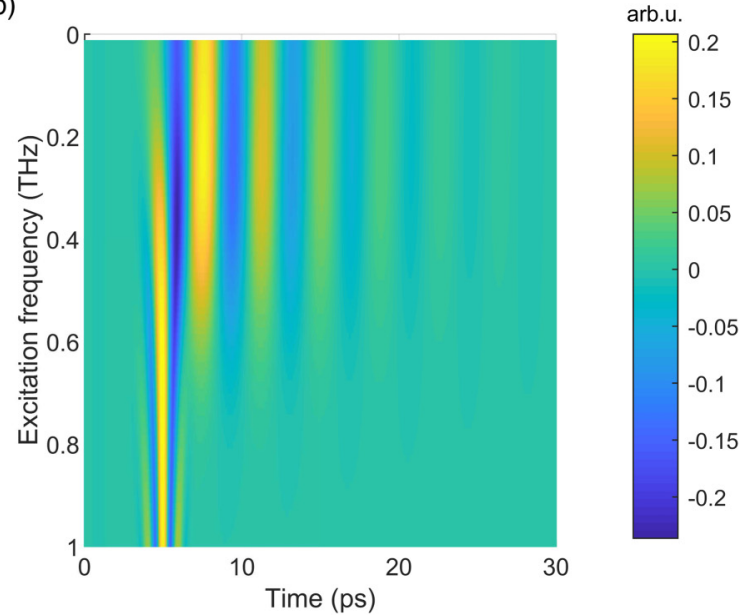

FIG. 4. 2D color plot of the terahertz temporal traces at different applied magnetic field maximum values from 0 to $1 \mathrm{~T}(\mathrm{a})$ and excitation frequencies from 0 to $1 \mathrm{THz}$ (b). 

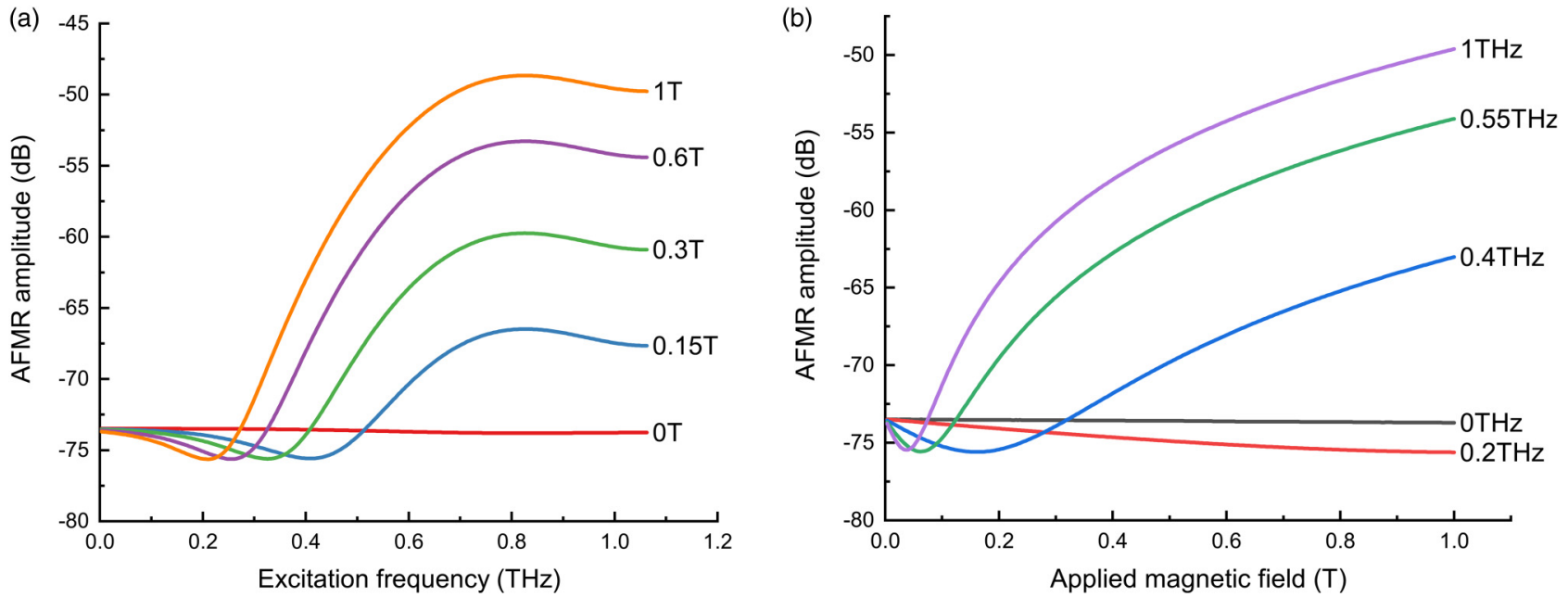

FIG. 5. Dependencies of the normalized response fundamental frequency spectrum peak on the (a) excitation frequency and (b) applied magnetic field.

$$
\alpha \omega_{e x} \Omega+\omega_{0}^{2} \frac{J_{1}^{2}\left(\beta_{1}\right)}{2} \sin \left(2 \beta_{0}\right)=0,
$$

where $\omega_{\max }=\gamma H_{\max } J_{n}\left(\beta_{1}\right)$ is the nth order Bessel function, $f(t)=$ $\exp \left[-\left(\frac{t-t_{0}}{\tau}\right)^{2}\right]$ is the envelope of a probing pulse, and $f^{\prime}(t)=d f(t) / d t$. The result of solving the averaged equations (5a) and (5b) in terms of the output electric field is shown in Fig. 3(b) in comparison with the solution of the initial equation (2). Thus, the averaged equation characterizing the envelope is an adequate approximation of the original model (2). Figure 3(c) shows the spectra of the pump terahertz signal and output response.

\section{SIMULATION RESULTS}

In response to the application of a constant magnetic field to the structure, the magnetic moments are displaced slightly from their equilibrium states. In turn, the spins undergo damped oscillations toward their initial state. The amplitude of these nonlinear oscillations strongly depends on the amplitude and frequency of excitation [see Figs. 4(a) and 4(b)]. Increasing the amplitude of the constant magnetic field results in the oscillations being observed more clearly, and after the amplitude of the magnetic field reaches $0.6 \mathrm{~T}$, the sample's response becomes similar to the waveform of the excitation. Changes in the excitation frequency on the other hand affect the response differently: the response pulse has exactly the same frequency as the excitation pulse.

The amplitude of the AFM precession also depends on both the amplitude of the constant magnetic field and the exact frequency of the terahertz excitation [see Figs. 5(a) and 5(b)]. An increase of the excitation frequency results in a nonlinear behavior of the amplitude of AFM precession such that harmonics are observed in the outgoing wave. This growth has a peak point after which the amplitude starts to slowly decrease.
The amplitude of the fundamental frequency also increases nonlinearly with an increase of the amplitude of the input terahertz pulse.

Figure 6 shows the dependency of the total obtained spectrum as a function of the frequency of external excitation for the amplitude of the constant magnetic field $H_{\max }=0.15 \mathrm{~T}$. It is clearly observable that, depending on the excitation frequency, a harmonic could be excited in the sample supplementary to the AFM resonance mode. The frequency of this additional harmonic is exactly the same as the frequency of the external electromagnetic wave and is not the eigenfrequency of the magnetic system.

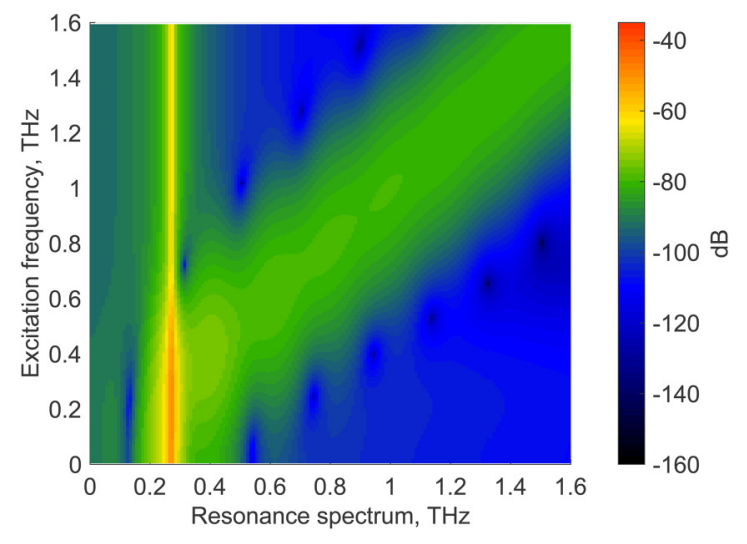

FIG. 6. The dependence of the spectrum of the excited AFM precession on the central frequency of excitation. Applied magnetic field is $H_{\max }=0.15 \mathrm{~T}$. 


\section{CONCLUSION}

In this work, we have theoretically demonstrated that a bilayered AFM-based heterostructure can be used as a nonlinear terahertzfrequency emitter, where the amplitude of the output excitation on the fundamental frequency varies nonlinearly with the amplitude of the pump pulse. We demonstrate control of the Néel vector trajectory in the AFM by tuning the peak amplitude and frequency of the terahertz pulse. We determine the bands of nonlinear excitation in a wide range of applied magnetic pulse amplitude using Fourier spectra. The proposed mathematical model and averaging method can be used for the description of nonlinear dynamics for a wide class of antiferromagnets and ferrimagnets. ${ }^{32}$ The obtained results could become crucial for the development of terahertz-frequency nanosized emitters and electromagnetic oscillators.

\section{SUPPLEMENTARY MATERIAL}

See the supplementary material for more detailed information on the two-parameter dependency of the resonance spectrum on both the excitation frequency and the constant magnetic field.

\section{ACKNOWLEDGMENTS}

Financial support from the Government of the Russian Federation (Agreement No. 074-02-2018-286) within the laboratory "Terahertz spintronics" of the Moscow Institute of Physics and Technology (State University) and the Russian Foundation for Basic Research (Project Nos. 18-57-76001, 18-37-20048, 18-29-27020, 18-29-27018, and 18-07-00485) is acknowledged. We gratefully acknowledge the Nederlandse Organisatie voor Wetenschappelijk Onderzoek (NWO-I) for their financial contribution, including the support of the FELIX Laboratory.

\section{REFERENCES}

${ }^{1}$ P. Němec, M. Fiebig, T. Kampfrath, and A. V. Kimel, "Antiferromagnetic optospintronics," Nat. Phys. 14, 1 (2018).

${ }^{2}$ T. Kampfrath, A. Sell, G. Klatt, A. Pashkin, S. Mährlein, T. Dekorsy, M. Wolf, M. Fiebig, A. Leitenstorfer, and R. Huber, "Coherent terahertz control of antiferromagnetic spin waves," Nat. Photonics 5, 31 (2011).

${ }^{3}$ S. Baierl, J. H. Mentink, M. Hohenleutner, L. Braun, T.-M. Do, C. Lange, A. Sell, M. Fiebig, G. Woltersdorf, T. Kampfrath et al. "Terahertz-driven nonlinear spin response of antiferromagnetic nickel oxide,” Phys. Rev. Lett. 117, 197201 (2016).

${ }^{4}$ R. Khymyn, I. Lisenkov, V. Tiberkevich, B. A. Ivanov, and A. Slavin, "Antiferromagnetic THz-frequency Josephson-like oscillator driven by spin current," Sci. Rep. 7, 43705 (2017)

${ }^{5}$ O. Sulymenko, O. Prokopenko, V. Tiberkevich, A. Slavin, B. Ivanov, and R. Khymyn, "Terahertz-frequency spin Hall auto-oscillator based on a canted antiferromagnet," Phys. Rev. Appl. 8, 064007 (2017).

${ }^{6}$ A. Kirilyuk, A. V. Kimel, and T. Rasing, "Laser-induced magnetization dynamics and reversal in ferrimagnetic alloys," Rep. Progress Phys. 76, 026501 (2013).

${ }^{7}$ P. S. Keatley, V. Kruglyak, P. Gangmei, and R. Hicken, "Ultrafast magnetization dynamics of spintronic nanostructures," Philos. Trans. R. Soc. A Math. Phys. Eng. Sci. 369, 3115-3135 (2011)

${ }^{8}$ J. Železný, H. Gao, K. Výborný, J. Zemen, J. Mašek, A. Manchon, J. Wunderlich, J. Sinova, and T. Jungwirth, "Relativistic néel-order fields induced by electrical current in antiferromagnets," Phys. Rev. Lett. 113, 157201 (2014).

${ }^{9}$ A. Kimel, B. Ivanov, R. Pisarev, P. Usachev, A. Kirilyuk, and T. Rasing, "Inertia-driven spin switching in antiferromagnets," Nat. Phys. 5, 727 (2009).
${ }^{10} \mathrm{P}$. Wadley, B. Howells, J. Železnỳ, C. Andrews, V. Hills, R. P. Campion, V. Novák, K. Olejník, F. Maccherozzi, S. Dhesi et al. "Electrical switching of an antiferromagnet," Science 351, 587-590 (2016).

${ }^{11}$ T. Jungwirth, X. Marti, P. Wadley, and J. Wunderlich, "Antiferromagnetic spintronics," Nat. Nanotechnol. 11, 231 (2016).

${ }^{12}$ D. Bossini, S. Dal Conte, Y. Hashimoto, A. Secchi, R. Pisarev, T. Rasing, G. Cerullo, and A. Kimel, "Macrospin dynamics in antiferromagnets triggered by sub-20 femtosecond injection of nanomagnons," Nat. Commun. 7, 10645 (2016).

${ }^{13}$ K. Olejník, V. Schuler, X. Martí, V. Novák, Z. Kašpar, P. Wadley, R. P. Campion, K. W. Edmonds, B. L. Gallagher, J. Garcés et al. "Antiferromagnetic cumnas multi-level memory cell with microelectronic compatibility," Nat. Commun. 8, 15434 (2017)

${ }^{14}$ T. Seifert, S. Jaiswal, U. Martens, J. Hannegan, L. Braun, P. Maldonado, F. Freimuth, A. Kronenberg, J. Henrizi, I. Radu et al. "Efficient metallic spintronic emitters of ultrabroadband terahertz radiation," Nat. Photonics. 10, 483 (2016).

${ }^{15}$ G. Torosyan, S. Keller, L. Scheuer, R. Beigang, and E. T. Papaioannou, "Optimized spintronic terahertz emitters based on epitaxial grown $\mathrm{Fe} / \mathrm{Pt}$ layer structures,” Sci. Rep. 8, 1311 (2018).

${ }^{16}$ D. Yang, J. Liang, C. Zhou, L. Sun, R. Zheng, S. Luo, Y. Wu, and J. Qi, "Powerful and tunable thz emitters based on the fe/pt magnetic heterostructure," Adv. Opt. Mater. 4, 1944-1949 (2016).

${ }^{17}$ M. Battiato, K. Carva, and P. M. Oppeneer, "Superdiffusive spin transport as a mechanism of ultrafast demagnetization," Phys. Rev. Lett. 105, 027203 (2010).

${ }^{18}$ E. Saitoh, M. Ueda, H. Miyajima, and G. Tatara, "Conversion of spin current into charge current at room temperature: Inverse spin-Hall effect," Appl. Phys. Lett. 88, 182509 (2006)

${ }^{19}$ Y. Tserkovnyak, A. Brataas, and G. E. W. Bauer, "Enhanced gilbert damping in thin ferromagnetic films," Phys. Rev. Lett. 88, 117601 (2002).

${ }^{20}$ A. Azevedo, L. Vilela Leão, R. Rodriguez-Suarez, A. Oliveira, and S. Rezende, "dc effect in ferromagnetic resonance: Evidence of the spin-pumping effect?," J. Appl. Phys. 97, 10C715 (2005).

${ }^{21}$ H. Jiao and G. E. W. Bauer, "Spin backflow and ac voltage generation by spin pumping and the inverse spin Hall effect," Phys. Rev. Lett. 110, 217602 (2013).

${ }^{22} \mathrm{O}$. Johansen and A. Brataas, "Spin pumping and inverse spin Hall voltages from dynamical antiferromagnets," Phys. Rev. B 95, 220408 (2017).

${ }^{23}$ R. Cheng, J. Xiao, Q. Niu, and A. Brataas, "Spin pumping and spin-transfer torques in antiferromagnets," Phys. Rev. Lett. 113, 057601 (2014).

${ }^{24}$ I. Dzyaloshinsky, "A thermodynamic theory of "weak" ferromagnetism of antiferromagnetics,” J. Phys. Chem. Solids 4, 241-255 (1958)

${ }^{25}$ T. Moriya, "Anisotropic superexchange interaction and weak ferromagnetism," Phys. Rev. 120, 91 (1960).

${ }^{26} \mathrm{~B}$. Ivanov, "Spin dynamics of antiferromagnets under action of femtosecond laser pulses," Low Temp. Phys 40, 91-105 (2014).

${ }^{27}$ E. Turov, A. Kolchanov, V. Men'shenin, I. Mirsaev, and V. Nikolaev, Symmetry and Physical Properties of Antiferromagnets (Fizmatlit, Moscow, 2001)

${ }^{28}$ S. A. Gulbrandsen and A. Brataas, "Spin transfer and spin pumping in disordered normal metal-antiferromagnetic insulator systems," Phys. Rev. B 97, 054409 (2018).

${ }^{29}$ J. Walowski and M. Münzenberg, "Perspective: Ultrafast magnetism and $\mathrm{THz}$ spintronics," J. Appl. Phys. 120, 140901 (2016).

${ }^{30}$ A. H. Morrish, Canted Antiferromagnetism: Hematite (World Scientific, 1994). ${ }^{31}$ H. Nakayama, K. Ando, K. Harii, T. Yoshino, R. Takahashi, Y. Kajiwara, K.-I. Uchida, Y. Fujikawa, and E. Saitoh, "Geometry dependence on inverse spin Hall effect induced by spin pumping in Ni 81 Fe 19/Pt films," Phys. Rev. B 85, 144408 (2012).

${ }^{32}$ R. Mikhaylovskiy, E. Hendry, A. Secchi, J. H. Mentink, M. Eckstein, A. Wu, R. Pisarev, V. Kruglyak, M. Katsnelson, T. Rasing et al. "Ultrafast optical modification of exchange interactions in iron oxides," Nat. Commun. 6, 8190 (2015) 